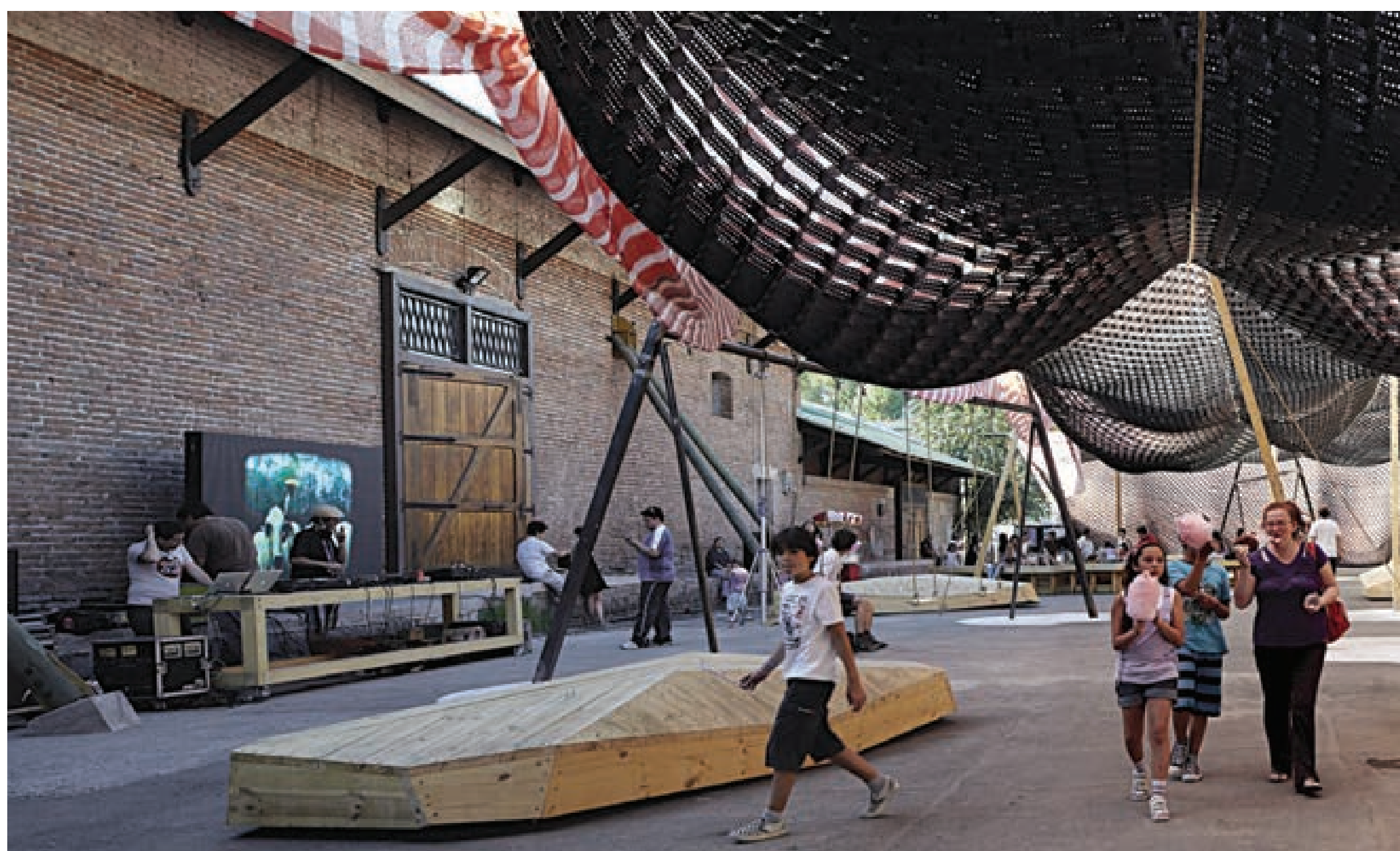

\title{
Eduardo Castillo
}

Profesor, Universidad de Talca, Talca

Arquitecto, Pontificia Universidad Católica de Chile, 2000.

Sus obras han sido publicadas y expuestas en Alemania,

España, Inglaterra, Italia, Japón y México, entre otros países.

Ha sido invitado a eventos y conferencias internacionales por universidades y Colegios de Arquitectos. Actualmente trabaja junto al arquitecto Smiljan Radic y es profesor de Taller de proyectos en la Escuela de Arquitectura de la Universidad de Talca. 
ARQUITECTO EDUARDO CASTILLO, 2010

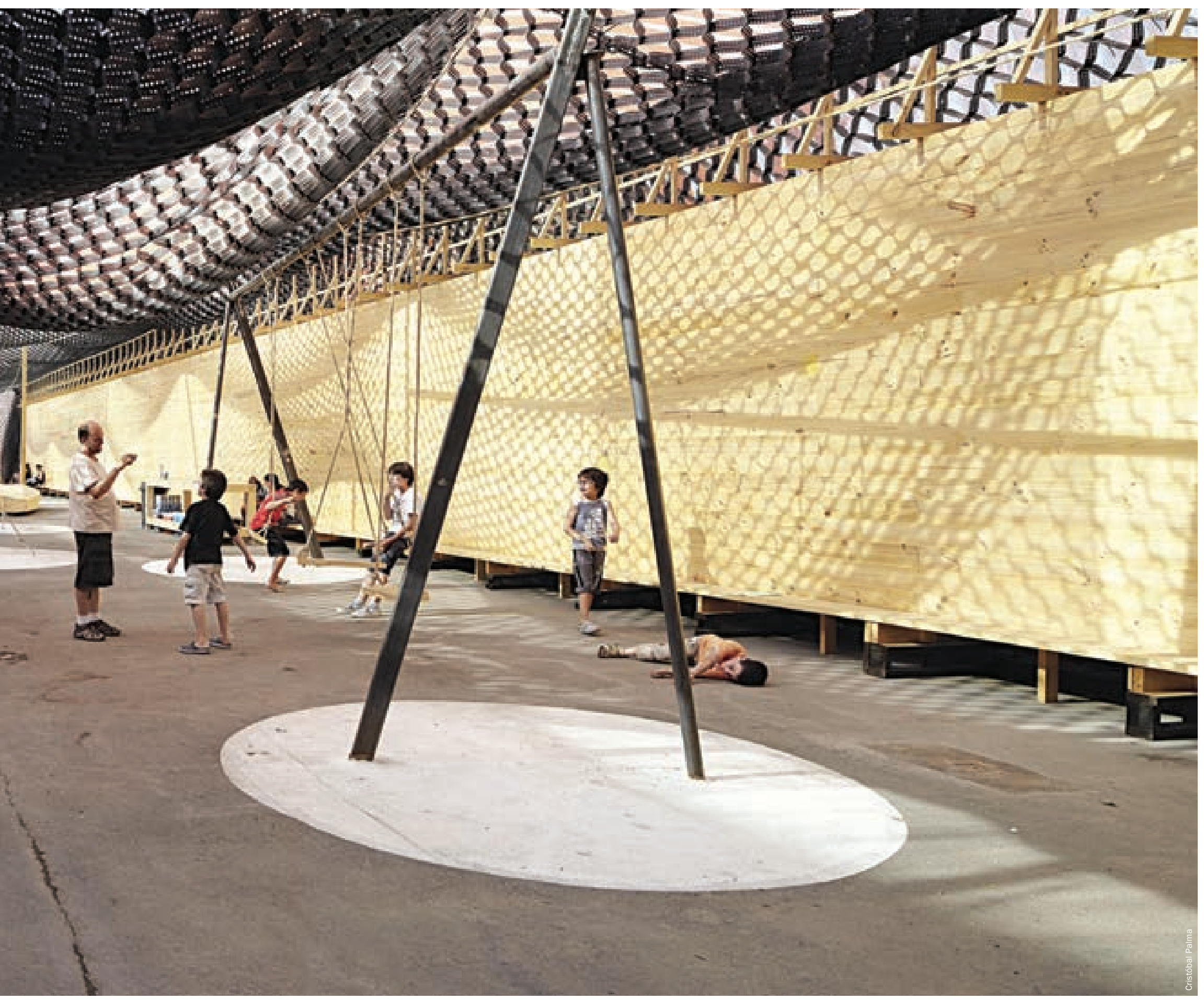

Siguiendo un modelo iniciado en Nueva York en 2000 por MOMA-PS1, esta es la primera experiencia en Sudamérica de una iniciativa que promueve el trabajo de nuevas generaciones de arquitectos a través de una construcción. Se trata de una instalación que, durante un mes en el verano, funciona como escenario templado para conciertos y juegos en la ciudad; esta vez, una cubierta catenaria e informe provee un ambiente de sombras y pesos en suspensión.

PALABRAS CLAVE Arquitectura-Chile, arquitectura efímera, madera, cubiertas, espacio público, mobiliario urbano

This is a program created by MOMA-PS1 in 2000 in New York. In its first South American version, the initiative aims to promote young talent through construction: during summer, the installation hosts music performances and open-air activities. This time it was a formless, hanging tent and its shadowy interior.

KEYWORDS Architecture-Chile, ephemeral architecture, wood, tent, public space, urban furniture 

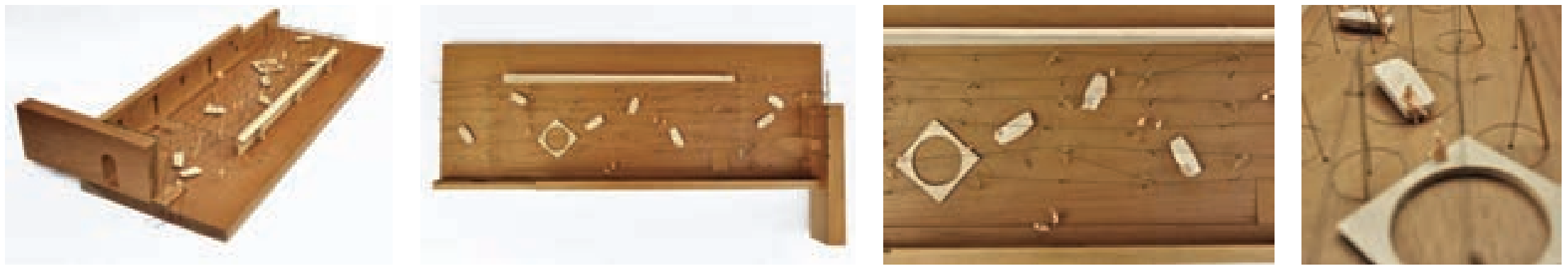

1

Esta es la construcción de un paisaje temporal, como muchos que comienzan a levantarse desde las fiestas de la primavera en valle central. Buscando templar los ambientes festivos, dan como resultado unas grandes atmósferas tamizadas de luz para celebrar independencias, santos, vírgenes, aniversarios, etc.

2

Así como las buenas cosas sirven para más de una función, las construcciones agrícolas casi siempre me han servido como punto de partida. Su lógica es la de una "buena construcción".

3

Creo que una buena arquitectura, primero tiene que ser una buena construcción -tan imperfecta como sea posible sostener-.

El encargo del concurso nos pedía lugares de sombra, lugares con agua y bancas para sentarse. Para eso, propuse que cada cosa no fuera entregada de manera unívoca, más bien lograran disolverse de manera confusa.

Que la sombra fuera coloreada y conformara todo el lugar mediante dos cubiertas: un gran manto tejido que permita tamizar la luz y que, a la vez, se deja caer de forma elástica, conformando una topografía de gran sinuosidad. Sobre ella, una cubierta más dinámica y coloreada.

Estos dos gigantescos manteles al sumarse generarán unas largas burbujas de aire, y permitirán templar medianamente la temperatura y, a la vez, fabricarán un tatuaje colorido que se imprimirá en todas las superficies que resistan su reflejo.

5

El agua aparecerá en estado líquido, contenida en una pileta que permita sumergir los pies sentados sobre una plataforma como soporte.

6

Finalmente para sentarse en un lugar medianamente cómodo -sirve cualquier cosa- en este caso siete macizos de madera facetada contribuyen a consolidar el "paisaje de simulación" propuesto.

Junto a ellos se suman doce columpios que se toman de la estructura tensada con palos y cuerdas -que es la principal estructura de soporte-.

7

Quizás solo una gran escenografía para unas fiestas musicales que no celebran nada, en medio de ninguna parte.

8

Aldo Rossi sugería en su libro Autobiografía científica, “...que para alcanzar su grandeza, la arquitectura debe ser olvidada o construir tan solo una imagen de referencia confundida con los recuerdos". Espero que todo este esfuerzo sea ese recuerdo del que habla Rossi,

...al menos ese es el deseo. ARQ

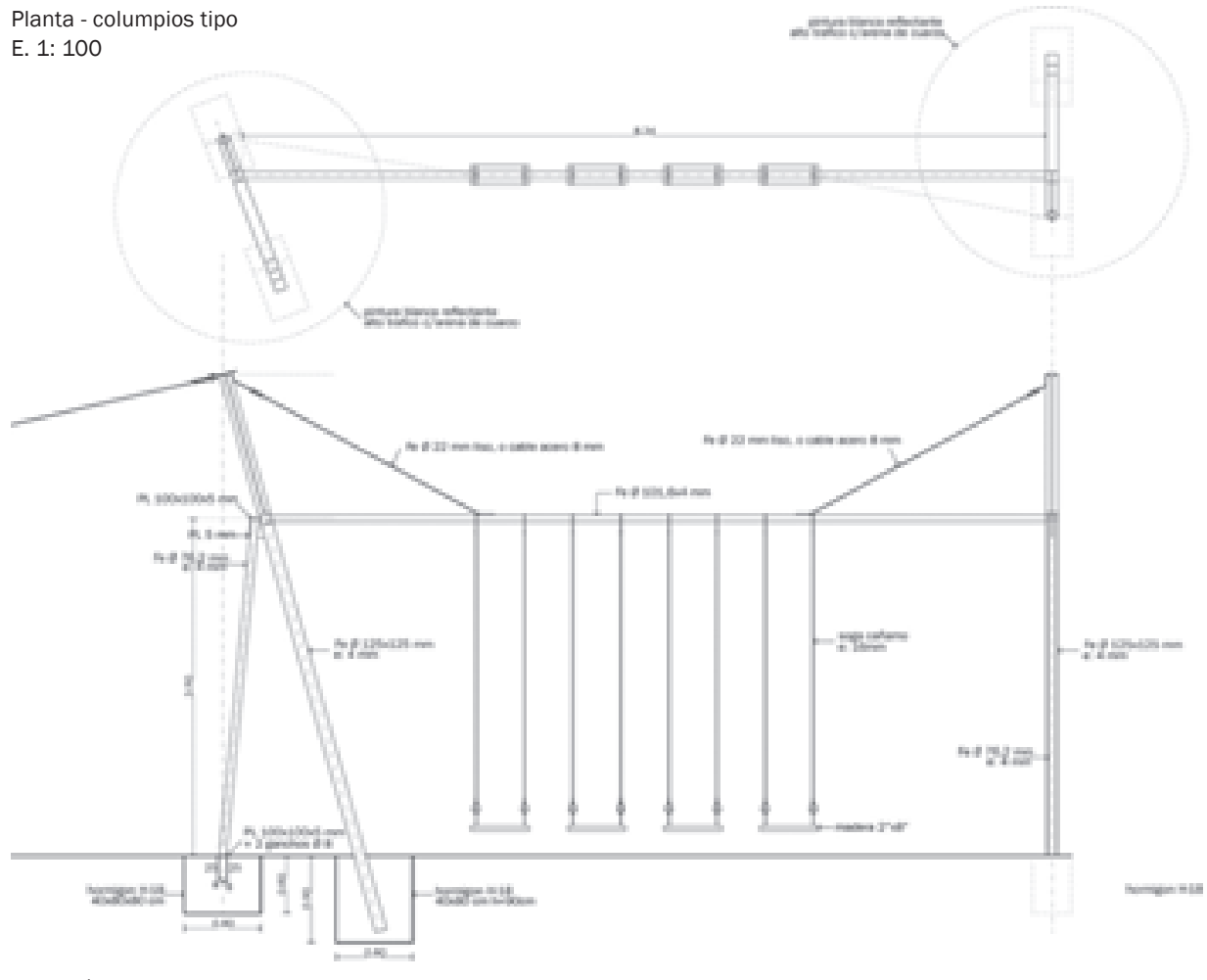

Elevación - columpios tipo

E. 1: 100

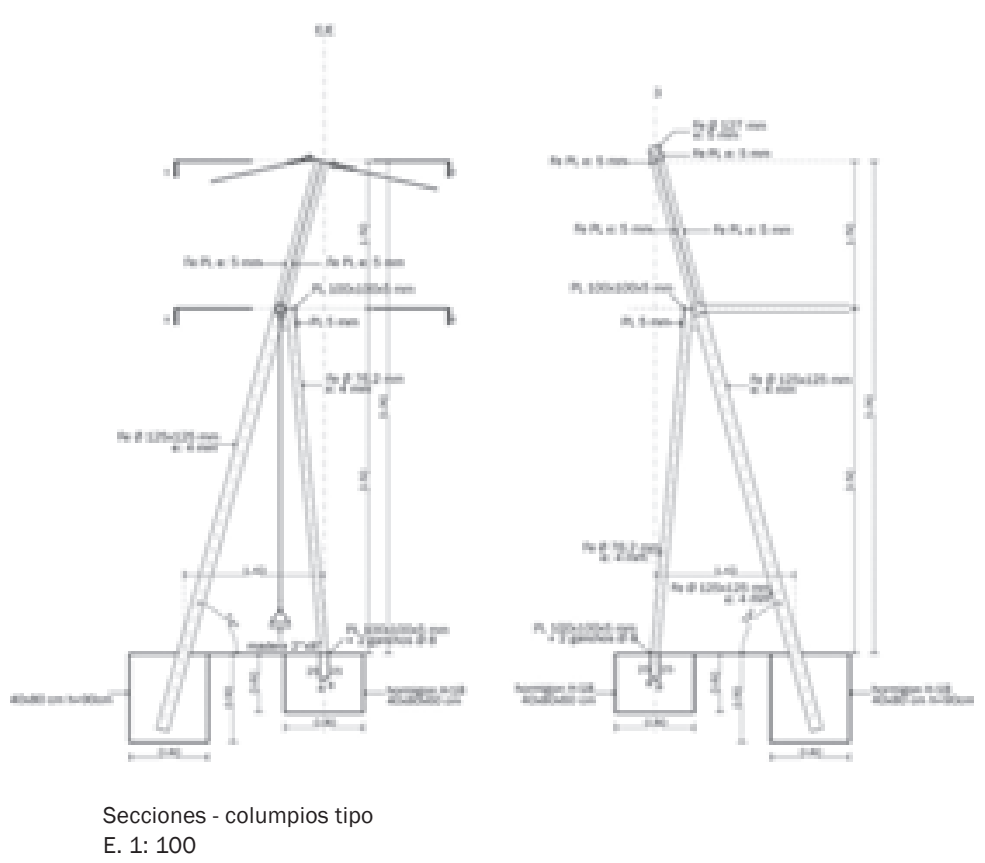



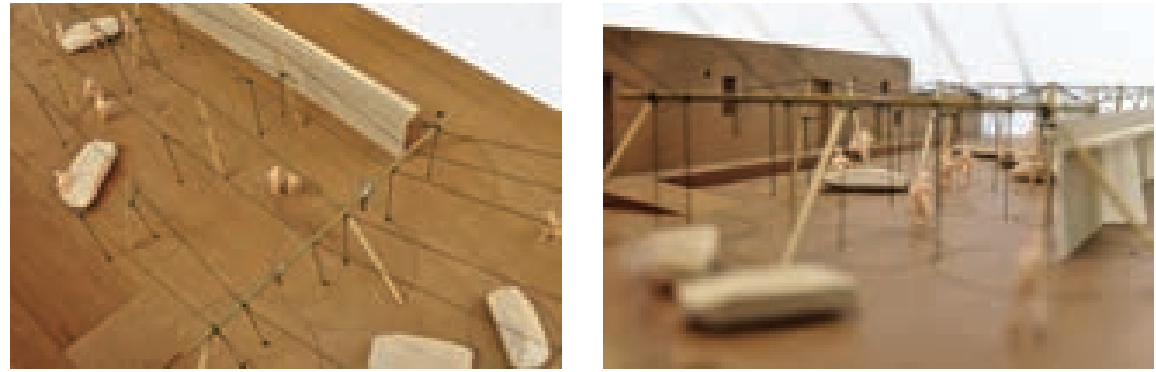

Corte longitudinal AA

E. 1: 500

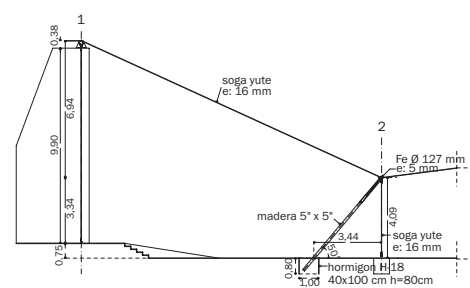

Planta piscina nivel intermedio $+0,20 \mathrm{~m}$

E. 1: 100
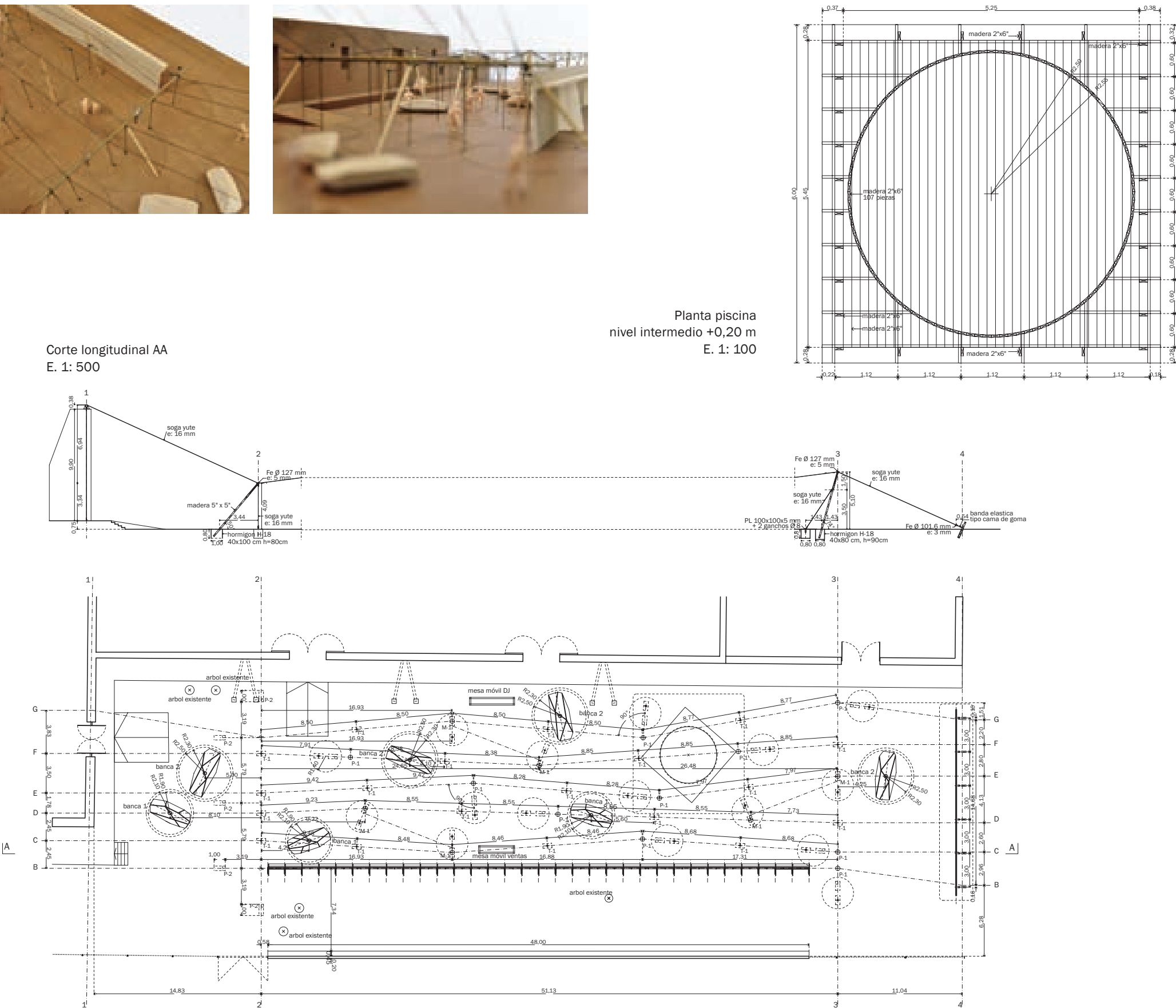

Planta general

E. 1: 500

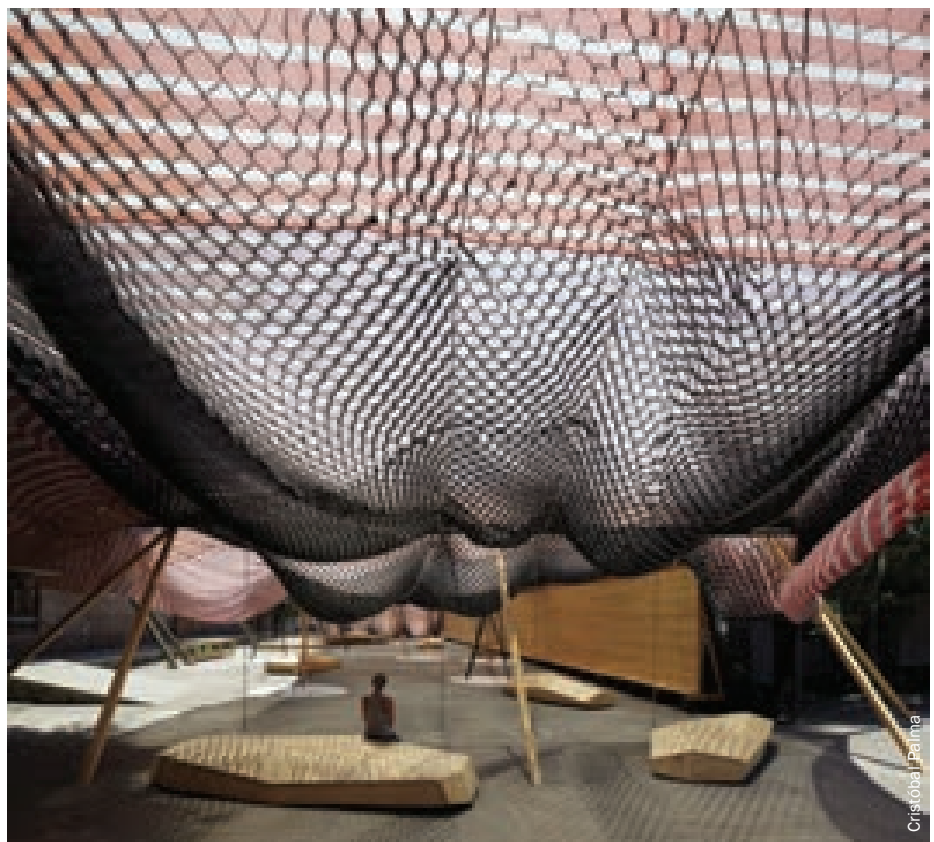

Young Architects Program YAP cONSTRUCTO 2010 / Arquitecto: Eduardo Castillo / Arquitectos asociados y colaboradores: María Francisca Navarro, Gonzalo Torres / Ubicación: Centro Cultural Matucana 100, Santiago / Cliente: CONSTRUCTO / Cálculo: Patricio Bertholet / Construcción: Rodrigo Gil, Juan Castillo, Nicolás González / Materiales predominantes: puntales de madera de 5" x 5" en maderas HILAM, pino radiata ARAUCO, soga de yute de $16 \mathrm{~mm}$, pletinas y anclajes metálicos de acero, malla raschel, cubierta flexible ENVIROGRID EGA203P de Emin Sistemas geotécnicos, pintura blanca reflectante / Superficie construida: $1.500 \mathrm{~m}^{2}$ / Año de proyecto: 2010 / Fecha de construcción: diciembre de 2010 / Fotografía: Cristóbal Palma, Luis Iturra

\section{Bibliografía sugerida}

CASTILLO, Eduardo. "Casa Gallinero, Casa 3 castaños". ARQ № 77 , Emergencia y materia. Ediciones ARQ, Santiago, abril de 2011. pp. 44-53.

CASTILLO, Eduardo. "Desde una memoria hecha de material". ARQ $N^{\circ} 51$, El sur de América. Ediciones ARQ, Santiago, julio de 2002, pp. 38-43.

CASTILLO, Eduardo. Conversaciones informales. Luis Izquierdo / Germán del Sol. Ediciones ARQ, Santiago, 2009.

http://arqecastillo.blogspot.com/

http://www.moma.org/interactives/exhibitions/yap/santiago.html 